Doug Geisler, Eva K. Grebel, and Dante Minniti, eds.

\title{
Extragalactic Globular Cluster Systems in the Near-IR
}

Thomas H. Puzia

Sternwarte der Universität München, Scheinerstr. 1, D-81679 München, Germany

Markus Kissler-Patig

European Southern Observatory, Garching, Germany

Jean Brodie

UCO/Lick Observatory, Santa Cruz, USA

Paul Goudfrooij

STScI, Baltimore, USA

Michael Hilker

Sternwarte der Universität Bonn, Germany

Dante Minniti

Pontificia Universidad Católica, Santiago, Chile

Steve Zepf

Michigan State University, East Lansing, USA

\begin{abstract}
Extragalactic Globular Clusters are useful tracers of galaxy formation and evolution. Photometric studies of globular cluster systems beyond the Local Group are still the most popular method to investigate their physical properties, such as their ages and metallicities. However, the limitations of optical photometry are well known. The better wavelength sampling of the underlying cluster's SED using $K$-band photometry combined with optical passbands allows us to create colors which reduce the age-metallicity degeneracy to the largest extent. Here we report on the very first results of our near-IR photometric survey of globular cluster systems in early-type galaxies outside the Local Group.
\end{abstract}

\section{Introduction}

Since the early 80 s several photometric studies of globular cluster systems have been performed in the near-infrared light out to $\sim 4 \mu \mathrm{m}$ and resulted in a better understanding of the metallicity of the observed stellar populations. However, 


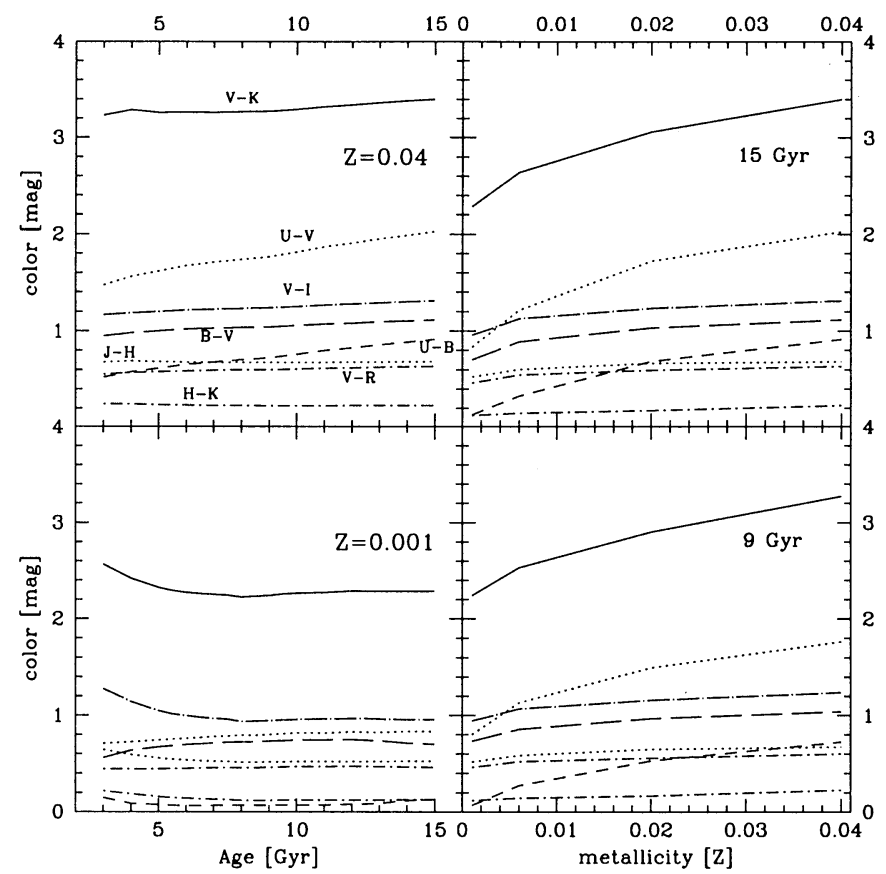

Figure 1. Broad-band colors as a function of age and metallicity for simple stellar populations (SSPs, here taken from Maraston 1998) parameterized by metallicity and age, respectively. Note the enhanced metallicity sensitivity for the $V-K$ color (solid line in the right panels) which shows weak age dependence for very old populations (left panels).

due to poor quantum efficiency of the array detectors all studies were confined to globular cluster systems of Local Group galaxies (M31: Frogel, Persson, \& Cohen 1980, Bonoli et al. 1987, 1992; Cohen \& Matthews 1994; LMC and SMC: Persson et al. 1983; M33: Cohen, Persson, \& Searle 1984; Fornax dSph: Zinn \& Persson 1981, etc.). With the most recent generation of near-IR detectors (such as the ISAAC instrument on the VLT) globular cluster systems outside the Local Group (e.g. NGC 7252: Maraston et al. 2001) have for the first time become accessible to deep K-band photometry which allows the study of clusters down to the turn-over of the luminosity function. Simultaneously, the number of accessible galaxies increased significantly making a larger near-IR photometric survey of their globular cluster systems feasible. Our group built up a homogeneous optical/near-IR database of a dozen extragalactic globular cluster systems in early-type galaxies to study their ages and mean metallicities, as well as of sub-populations within the systems. 


\section{Why do we study globular clusters in the near-infrared?}

The combination of near-IR and optical broad-band photometry generates highly metallicity-sensitive colors compared with colors constructed from optical passbands only. Such a metallicity-sensitive color is e.g. $V-K$. While the $V$ filter samples mainly the light from the turn-off population in a globular cluster, the $K$ band is most sensitive to the light emitted by cooler giant-branch stars. For old stellar populations (like in globular clusters) the turn-off is relatively stable in color and magnitude as a function of age and metallicity (see e.g. Yi et al. 2001). Simultaneously, the luminosity of the giant branch (at a given $T_{\text {eff }}$ ) is metallicity sensitive (e.g. Saviane et al. 2000). It is thus interesting to combine colors such as the $V$ and $K$ passbands to create a predominantly metallicity-sensitive photometric color for old stellar populations.

Figure 1 shows the change of some broad-band colors as a function of age and metallicity. Note the relatively flat curve for $V-K$ as a function of age (left panels) in combination with the steep slope as a function of metallicity (right panels) for very old stellar populations. $V-K$, constructed from broadband optical and near-IR filters, is thus one of the best photometric "metallicity indicators" among the current possible combinations. $V-K$, in combination with a more age-sensitive color, can be used to reduce the well-known agemetallicity degeneracy of photometric indicators.

From a purely practical point of view, it also presents the advantage that $V$ and $I$ are available for a great number of galaxies in the WFPC2 archive, and only the $K$ band needs to be acquired. Further, the ground-based facilities can reach $K$ band magnitudes equivalent to the HST optical ones in very similar field of views.

\section{Globular Cluster Color Distributions, $V-I$ vs. $V-K$}

The bi- and multi-modality of globular cluster color distributions is thought to be due to an unknown mix of age and mean metallicity differences between the main globular cluster sub-populations (see also the contribution of KisslerPatig in this volume and Fig. 1). The $V-K$ color provides a more accurate estimate of the underlying metallicity distribution of a globular cluster system. Another useful feature of the $V-K$ color is its larger dynamic range in terms of metallicity (see Figure 2) when compared to that of e.g. the $V-I$ color.

Figure 2 shows the comparison of the $V-I$ and $V-K$ color distribution of globular clusters in NGC 3115 (isolated S0 galaxy) and NGC 4365 (group giant elliptical, E3). In the cases of NGC 3115 and NGC 4365 we found 77 and 138 globular cluster candidates, respectively. The $V_{\mathrm{F} 555 \mathrm{~W}}, I_{\mathrm{F} 814 \mathrm{~W}}$, and $K$ photometry was obtained with WFPC2 on board HST and ISAAC on the VLT. The scale dilatation going from the $V-I$ distribution to $V-K$ distribution is apparent. The solid lines are probability-density function (PDF) estimates of the underlying color distributions (using an Epanechnikov kernel, see Silverman 1986). The PDF technique provides also continuous significance estimates which are shown as dashed lines and mark the $1 \sigma$ confidence limits of the PDF estimate. Vertical lines show the best mean color estimates using the KMM code (Ashman et al. 1994) which in the case of $V-I$ were taken from Larsen 

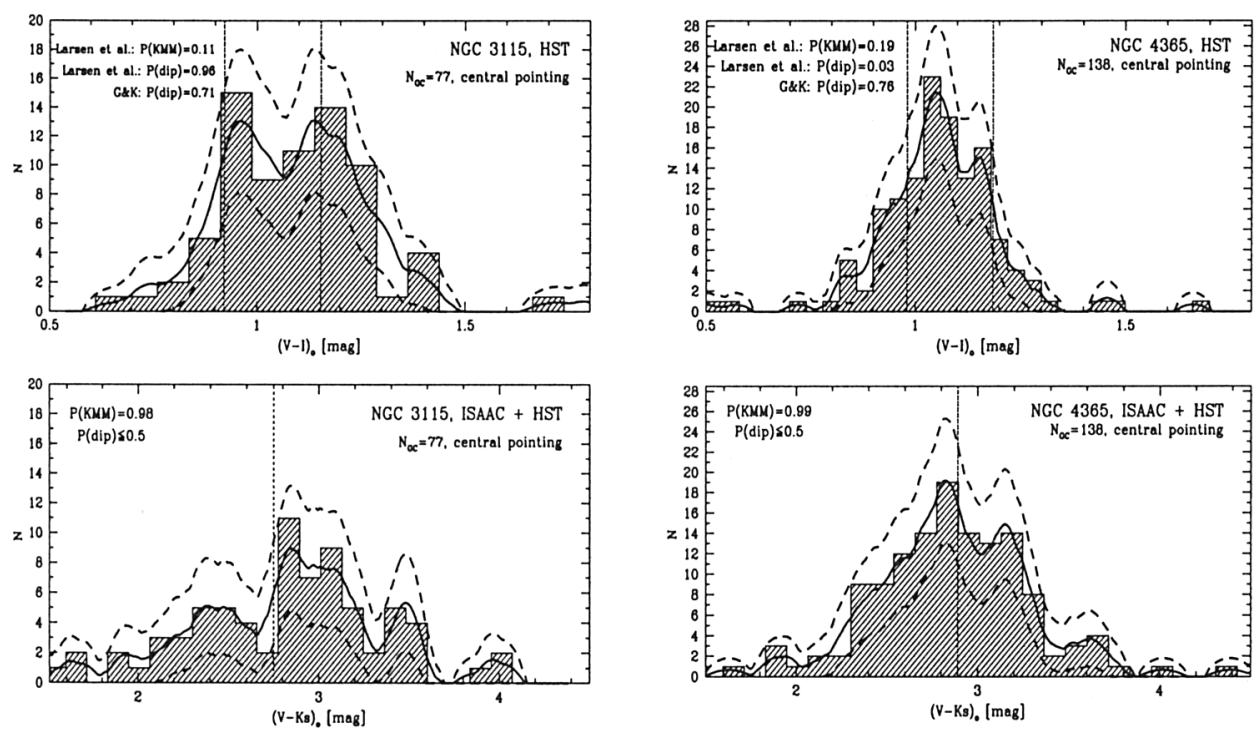

Figure 2. $\quad(V-I)$ and $(V-K)$ color distributions of globular clusters in NGC 3115 (left panel) and NGC 4365 (right panel).

et al. (2001) and re-measured for the $V-K$ colors. The bimodality likelihood as given by $\mathrm{KMM}$ is indicated as $\mathrm{P}(\mathrm{KMM})$ in each panel. Numbers close to 0 indicate that a bimodal distribution is preferred over a single Gaussian. For the $V-K$ distribution we also re-measured the DIP statistics $(V-I$ values were taken from Larsen et al. 2001 and Gebhardt \& Kissler-Patig 1999) which quantifies the significance of a dip in the color distribution. A numerical value of $\mathrm{P}(\mathrm{dip})$ of less than 0.5 indicates that a dip is not very likely.

While in the case of NGC 3115 the $V-I$ bimodality disappears in the $V-K$ diagram which is mostly due to the small sample size the $V-K$ diagram of NGC 4365 starts to show a clearer separation of the inherent globular cluster sub-populations although the $1 \sigma$ limits easily allow a single-mode distribution.

\section{Color-Color Diagrams}

Figure 3 shows $(V-I)$ vs. $(V-K)$ diagrams for NGC 3115 and NGC 4365 along with published globular cluster data of the Milky Way, M31 (Barmby et al. 2000) and preliminary data of NGC 4478 and M87 (from Kissler-Patig, Brodie \& Minniti 2001, in prep.). As a guide to the eye two metallicity tracks for a 15 Gyr old simple stellar population have been included. The slightly bluer track was taken from Maraston (2001), the redder form Bruzual \& Charlot (2001). Age decreases as one moves perpendicular to the isochrones to the lower right. The metallicity ranges from $[\mathrm{Fe} / \mathrm{H}]=-2.25$ dex at the blue end to $+0.3 \mathrm{dex}$ at the red end. The photometric errors in both diagrams are $\lesssim 0.1 \mathrm{mag}$ in 

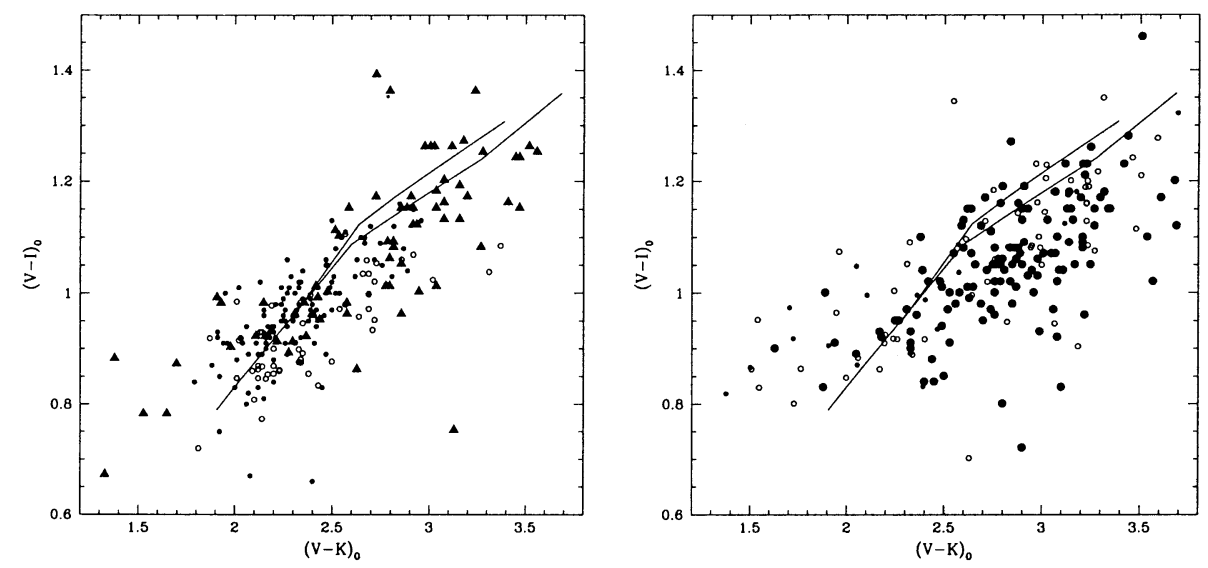

Figure 3. Color-color diagrams for NGC 3115 (left panel, filled triangles) and NGC 4365 (right panel, large filled dots). The left panel shows also globular clusters in the Milky Way (open circles) and in M31 (dots) while the right panel also includes preliminary globular cluster data of NGC 4478 (dots) and M87 (open circles) (taken from KisslerPatig, Brodie \& Minniti 2001 in prep.). All data are de-reddened.

$V-I$ and $\lesssim 0.15 \mathrm{mag}$ in $V-K$ and all datasets were corrected for foreground reddening.

The color-color diagram of NGC 3115 shows that the bimodality in the globular cluster color distribution (see Fig. 1) is mainly due to a difference in metallicity although a small age difference cannot be ruled out. The old Milky Way and M31 globular clusters (left panel of Fig. 3) coincide in color with the blue sub-population of NGC 3115 while the red sub-population appears to be more metal-rich than any of the globular clusters in the two Local Group spirals. In contrast to globular cluster systems of late-type and S0 galaxies the mean locus of globular clusters in early-type galaxies appears slightly redder by $\Delta(V-K) \approx+0.3 \mathrm{mag}$. The most likely reason for this shift is a combination of younger ages and higher metallicities of globular clusters in early-type galaxies. This will be quantified in a detailed comparison with available SSP models and will be published elsewhere (Puzia et al. 2002, in preparation). A rough estimate of the mean age and metallicity for the bulk of globular clusters in NGC 4365 at $V-I \approx 1.1$ and $V-K \approx 2.8$ allows an age of $\sim 3-6$ Gyr and $\sim 1 /{ }_{2} Z_{\odot}-2 Z_{\odot}$ metallicities. A similar result is found for the most metal-rich clusters in M87, but awaits to be quantified.

All this, of course, is based on the assumption that the SSP models are well calibrated in their high-metallicity parts which is not necessarily true since the number of high-metallicity and low- $T_{\text {eff }}$ stars in the local neighborhood which could serve as calibrators for stellar model atmospheres is small.

A comparison of $V-I$ and $V-K$ colors of intermediate-age $(\sim 0.3-1$ Gyr) solar-metallicity globular clusters, as they have been found in NGC 7252 (Maraston et al. 2001) with $0.6 \lesssim(V-I) \lesssim 0.7$ and $2.0 \lesssim(V-K) \lesssim 2.6$, and 
NGC 4365 globular clusters implies that the latter must be significantly older than $\sim 1$ Gyr. Very young globular clusters would also be noticeable in the globular cluster luminosity function as very bright objects.

The use of our database will allow to study the difference between globular clusters in late and early-type galaxies in detail and address the question how the environmental density of a host galaxy affects its globular cluster systems. The data will furthermore allow us to search for intermediate age globular clusters and to study the globular cluster luminosity function in great detail.

\section{References}

Ashman, K. A., Bird, C. M., \& Zepf, S. E. 1994, AJ, 108, 2348

Barmby, P., Huchra, J. P., Brodie, J. P., Forbes, D. A., Schroder, L. L., \& Grillmair, C. J. 2000, AJ, 119, 727

Bonoli, F., Fusi Pecci, F., Delpino, F., \& Federici, L. 1987, A\&A, 185, 25

Bonoli, F., Delpino, F., Federici, L., Fusi Pecci, F., \& Longmore, A. J. 1992, A\&AS, 96, 163

Bruzual A., G. \& Charlot, S. 2001, private communication

Cohen, J. G., Persson, S. E., \& Searle, L. 1984, ApJ, 281, 141

Cohen, J. G. \& Matthews, K. 1994, AJ, 108, 128

Frogel, J. A., Persson, S. E., \& Cohen, J. G. 1980, ApJ, 240, 785

Gebhardt, K. \& Kissler-Patig, M. 1999, AJ, 118, 1526

Larsen, S. S., Brodie, J. P., Huchra, J. P., Forbes, D. A., \& Grillmair, C. J. 2001, AJ, 121, 2974

Maraston, C. 1998, MNRAS, 300, 872

Maraston, C. 2001, in preparation

Maraston, C., Kissler-Patig, M., Brodie, J. P., Barmby, P., \& Huchra, J. P. 2001, A\&A, 370, 176

Persson, S. E., Aaronson, M., Cohen, J. G., Frogel, J. A., \& Matthews, K. 1983, ApJ, 266, 105

Saviane, I., Rosenberg, A., Piotto, G., \& Aparicio, A. 2000, A\&A, 355, 966

Silverman, B. W. 1986, Density Estimation for Statistics and Data Analysis, Monographs on Statistics and Applied Probability, Vol. 26, Chapman \& Hall

Yi, S., Demarque, P., Kim, Y.-C., Lee, Y.-W., Ree, C., Lejeune, Th., \& Barnes, S. 2001, ApJ, in press, astro-ph/0104292

Zinn, R. \& Persson, S. E. 1981, ApJ, 247, 849

\section{Discussion}

D. Alloin: There are now several groups computing SSP models (Padova, Bruzual \& Charlot, Maraston etc.). How do their predictions compare at a given age-metallicity point? 
T. Puzia: For all the SSP models we used, the low-metallicity regimes are in very good agreement for old and intermediate age populations. Model-to-model variations start to be apparent in the high-metallicity regions where most of the models lack accurate calibrators for old and young ages. Looking at the modelto-model discrepancies in the $V-I$ and $V-K$ color gives an age uncertainty of $\sim 2-4$ Gyr. Interestingly, the iso-metallicity tracks do not differ significantly, that is less than the photometric errors. It appears that age is more difficult to calibrate or is more dependent on the choice of calibrators than metallicity. However, with today's accuracy of near-IR photometry it would be premature to confirm or reject any SSP model. Once we manage to lower the photometric errors down to the level of model-to-model variations we can start to think about the validity of a given SSP model. It is, moreover, noteworthy to mention that at this precision we will start to decide about the variations in the intrinsic parameters of a stellar population (e.g. detailed chemical composition, stellar structural parameters, etc.).

P. Barmby: Do you have to worry about the difference between the standard $K$ band and $K_{s}$ ?

T. Puzia: The $K_{s}$ ("K-short") filter is a slightly modified $K$-band standard filter which was designed to reduce the thermal background for warm groundbased telescopes. It is different from the $K$ ' ("K-prime") filter in use at Mauna Kea Observatory. The filter transmission curve of $K_{s}$ drops before reaching the CO-band absorption at $\sim 2.4 \mu \mathrm{m}$ and thus avoids the impact of variations in the $\mathrm{CO}$ abundance on the photometric magnitude. The systematic corrections between $K$ and $K_{s}$ are of the order of a few $0.01 \mathrm{mag}$ while $K$-band magnitudes are brighter. 